\title{
Integrated Optical and Electronic Interconnect Printed Circuit Board Manufacturing
}

David R. Selviah*, F. Aníbal Fernández *, loannis Papakonstantinou*, Kai Wang*, Hadi Bagshiahi*, Andy C. Walker\#, Aongus McCarthy\#, Himanshu Suyal\#, David A. Hutt+, Paul P. Conway+, John Chappell+, Shefiu S. Zakariyah+, Dave Milward ${ }^{\circ}$

d.selviah@ee.ucl.ac.uk, a.c.walker@hwu.ac.uk,d.a.hutt@lboro.ac.uk, dave_milward@xyratex.com

*Department of Electronic and Electrical Engineering, University College London, UCL, UK

\#School of Engineering and Physical Sciences, Heriot Watt University, UK

+Wolfson School of Mechanical and Manufacturing Engineering, Loughborough University, UK

${ }^{\circ}$ Xyratex Technology Ltd., Havant, UK

\section{Structured Abstract}

Purpose - to introduce the Innovative Electronics Manufacturing Research Centre (leMRC) Flagship Project: Integrated Optical and Electronic Interconnect PCB Manufacturing (OPCB), its objectives, its consortium of 3 Universities and 10 companies and to describe the university research being carried out. This paper briefly reviews the motivation for developing novel polymer formulations, fabrication techniques, layout design rules and characterisation techniques for hybrid electronic and optical printed circuit boards using multimode polymer optical waveguide interconnects.

Design/methodology/approach - We are investigating a number of different fabrication techniques which we compare with each other and with modelled calculations waveguide components. The fabrication techniques include photolithography, laser ablation, direct laser writing, embossing, extrusion and ink jet printing.

Findings $-A$ number of design rules for polymer multimode waveguides have been found and published. Techniques for ink jetting polymer to print waveguides and laser ablation techniques have been developed. New formulations of polymer which cure faster for direct writing have also been developed.

Research limitations/implications - Further work is needed to thicken the ink jet printed polymer and to investigate side wall roughness of the ablated waveguides and development of new polymer formulations for dry film. Further research is also needed on construction of prototype system demonstrators.

Practical Implications - the fabrication techniques being developed are designed to be transferred to industrial PCB manufacturers to enable them to make higher value optical printed circuit boards. The design rules being discovered are being entered into commercial PCB layout software to aid designers of optical printed circuit boards.

Originality/value - The paper is of interest to $P C B$ manufacturers who wish to upgrade their processes to be able to manufacture optical printed circuit boards. The university research is original and some published to date in the publications in the reference list.

Keywords - Polymer, PCB manufacturing, PWB, Laser machining, Ink Jet Printing, Laser Ablation, Laser Writing, Photolithography, Manufacturing, Waveguide, Optical Interconnect

Paper type - Short paper 


\section{Biographies}

David R. Selviah is Senior Lecturer at Department of Electronic and Electrical Engineering, University College London, UCL. He came to UCL in 1987 after studying Physics and Theoretical Physics at Trinity College, Cambridge University, Engineering Science at Christchurch, Oxford University and 3 years industrial research on surface acoustic wave (SAW) adaptive correlator pulse compression radar filters. His research includes $10 \mathrm{~Gb} / \mathrm{s}$ multimode polymer waveguide OPCBs, tuneable microlenses and filters, holographic optical elements and storage, high sensitivity coherent detection, LCD backlights, higher order neural networks, pattern recognition and real-time image processing. $\mathrm{He}$ is a member of IOP, OSA, EOS and IEEE.

F. Aníbal Fernández received the BSc degree in Applied Mathematics fro the Universidad de Chile, Santiago, in 1969, and the PhD degree in Electrical Engineering from University College London, UK, in 1981. Until 1985, he was on the academic staff of the Department of Electrical Engineering Universidad. Chile. In 1986, he joined the staff of the Department of Electronic and Electrical Engineering, UCL where his is currently a Senior Lecturer. His research interests include microwave and optical aspects of electromagnetic theory, especially the use of numerical methods in those fields and in the last 12 years have concentrated mainly in the modelling of liquid crystal devices.

Ioannis Papakonstantinou graduated with a first class diploma in Electrical and Computer Engineering from the National Technical University in Athens (NTUA), Greece in 2000. After spending one year in the industry working on network management systems he moved to UCL, UK, to study for the MSc in Broadband Technologies for Communications under EPSRC funding, which he was awarded with distinction. Since 2003, he is working towards the completion of his PhD on optical backplane interconnections in the Optical Systems and Devices Lab at UCL, funded by Xyratex Ltd., and via an EPSRC doctoral training award. Ioannis was awarded a Center of Scientific Enterprises Limited (CSEL) bursary in 2007 and a Cannon Foundation in Europe fellowship also in 2007.

Kai Wang received his BSc in Electronic Engineering and Telecommunications from Shenzhen University, China in 2001, and his MSc in Microelectronic Systems and Telecommunications from Liverpool University in 2002 being awarded the International Student Scholarship. In 2003 he began $\mathrm{PhD}$ research at Department of Electronic and Electrical Engineering, UCL, becoming a research fellow in 2004. He has advanced skills in ray trace modelling of LCDs, backlights and multimode waveguides. His current research is to establish design rules for multimode waveguides by experiment and modelling. He was awarded the IET Hudswell International Research Scholarship and the Master Degree Research Scholarship.

Hadi Baghsiahi received his Bachelor Degree in Physics and Lasers from Shiraz University, Iran, in 2002. In the same year, he was awarded a student scholarship at the International Center for Science and High Technology \& Environment Sciences, Kerman, Iran. He was awarded an MSc degree in Photonics and Optical Telecommunications from Kerman University, in 2005. In October 2007, he joined the Department of Electronic and Electrical Engineering in UCL to start a PhD degree. $\mathrm{He}$ is currently researching into the design, modeling, and fabrication techniques for optical polymer waveguides integrated into optical printed circuit boards, OPCBs.

Prof. Andy C. Walker studied Physics at Essex University, where he received a BA, MSc and, in 1972, PhD on the observation of the photon drag effect in semiconductors. As an NRC Canada Postdoctoral Fellow he worked on the generation of picosecond pulses from $\mathrm{CO}_{2}$ lasers. Following eight years at Culham Laboratory, he joined Heriot-Watt University in 1983, where he is currently Professor of Modern Optics and Vice Principal. His research covers semiconductor optoelectronic devices and optical interconnects. Professor Walker is former IOP Scottish Branch Chairman and Vice President of the Royal Society of Edinburgh. He has published over 200 scientific papers.

Aongus McCarthy is from Ferbane, Co. Offaly, Ireland. He graduated with a BSc from University College Galway in 1989, a Diploma in Electronic Engineering from the Institute of Technology in Carlow, Ireland in 1990 and a BSc in Physical Optoelectronics from Essex University, England in 1991. He received a PhD degree in Physics from Heriot-Watt University, Edinburgh, Scotland, in 2002. His research interests include optical interconnects (free-space and guided-wave), optical and optomechanical design, direct laser-writing techniques and microscope systems. Dr. McCarthy is a member of the Optical Society of America and the IEEE Laser and Electro-Optics Society. 
Himanshu Suyal received his M.Sc. degree in Physics from Kumaon University, Nainital, India and an M.Tech. degree in Laser Science and its applications from D.A.V.V. Indore, India in 1999 and 2001 respectively. He then moved to Scotland to join the School of Engineering and Physical Sciences at Heriot-Watt University to study for a Ph.D. in the field of optical interconnects, which he completed in 2006. He is working as a Research Associate since October 2006 on the leMRC funded OPCB project. His major research interests are in guided-wave optical interconnects and direct laser-writing of microstructures in photopolymer.

David Hutt is a Senior Lecturer at Loughborough University, UK and a Senior Member of the IEEE. He received his $\mathrm{BSc}$ in Chemistry and $\mathrm{PhD}$ in Surface Science from Imperial College, London and then studied fundamental surface chemistry / physics as a Research Associate at a number of UK universities. In 1997, David joined the Interconnection Group at Loughborough University, applying this background to the field of electronics manufacture and was appointed to a lectureship in 1999. His research interests include high density interconnect, fluxless soldering and novel substrate manufacture.

Paul Conway is Professor of Manufacturing Processes at the Wolfson School of Mechanical and Manufacturing Engineering, Loughborough University, UK. He is also the Director of the UK's national Innovative Electronics Manufacturing Research Centre, established to allow industry to access and influence public funded academic research in the UK. He has previously worked for Fisher Body Overseas Corporation, GMC and as an NPL funded research engineer at Loughborough. He has been active in contract research, training and consultancy in electronics manufacture since 1990, holding substantive research awards from EPSRC, EC, DTI and industry and has published widely in this field. His interests lie in manufacturing processes, simulation, sensing, actuation and control and understanding of process-materials interactions.

John Chappell is a research fellow in the Wolfson School of Mechanical and Manufacturing Engineering, Loughborough University, UK, working on ink jet printing of polymer waveguides on printed circuit boards as part of the OPCB project consortium. His background is in graphic design.

Shefiu S. Zakariyah is currently studying for his PhD within the Wolfson School of Mechanical and Manufacturing Engineering, Loughborough University, UK, working on laser ablation for the fabrication of optical waveguides on printed circuit boards as part of the OPCB project consortium. His background is in general Engineering with emphasis in Electrical \& Electronic Engineering. $\mathrm{He}$ is a graduate member of the Institution of Engineering and Technology (IET) and a member of the Laser Institute of America (LIA) \& International Association of Engineers (IAENG).

Dave Milward, Development Manager, Xyratex Technologies Ltd. - Dave Milward received a BEng degree in electrical \& electronic engineering at Liverpool University, England. Before joining Xyratex he spent 25 years working for IBM Hursley, initially as a digital design engineer before moving into management. Since 1994 he has worked as product development manager for Networking and Storage systems and more recently as project manager for collaborative research projects involving academic and industrial partners. Mr Milward is a Chartered Engineer and member of the Institution of Engineering and Technology (IET) 


\section{Introduction}

At high bit rates copper tracks in printed circuit boards suffer severe loss and pulse distortion due to radiation of electromagnetic waves, dispersion and bandwidth limitations. The loss can be overcome to some extent by transmitting higher power pulses, by changing the dielectric constant and loss tangent of the PCB substrate material. However, high power pulses consume power and can cause electro-migration which reduces the board lifetime although the copper tracks can be surrounded by another metal to prevent this at the expense of further processing steps. The use of special board material can be costly and some materials containing high dielectric constant crystallites can cause poor adhesion. The pulse distortion, dispersion and bandwidth limitations can be overcome to some extent by the use of pulse pre-emphasis and adaptive equalisation at further cost. Electromagnetic waves are radiated efficiently at high bit rates removing power from the track so causing loss, but more importantly they are also received efficiently by other nearby and distant copper tracks on the same PCB, or on adjacent PCBs, or PCBs and other electrical conductors outside of the system enclosure. This EMI crosstalk causes increased noise and so degrades the signal to noise ratio and the bit error rate of the copper track interconnections. Therefore, the main forces driving the development of alternative interconnect technologies are the EMI crosstalk which becomes increasingly more serious as bit rates increase for longer and denser interconnects, and secondly the cost of overcoming the other problems that occur in copper interconnects at high bit rates.

Optical fibres have replaced copper cables for long distance, backbone and submarine applications where they offer wide bandwidths for low loss, produce and

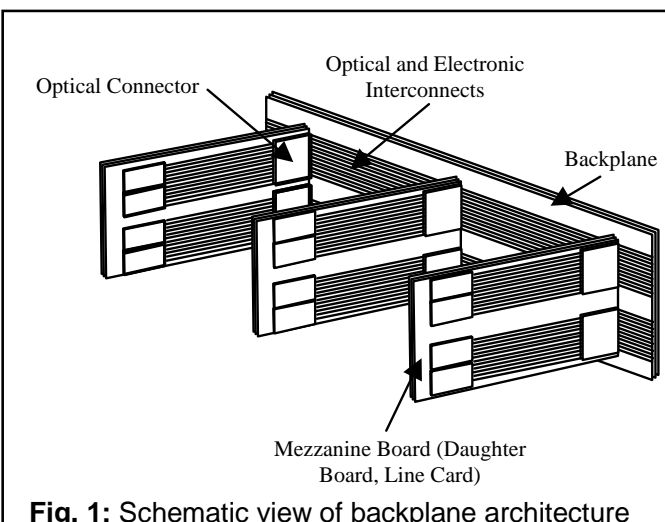

Fig. 1: Schematic view of backplane architecture

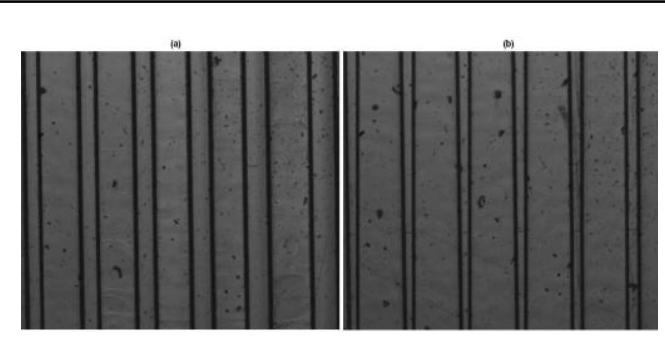

Fig. 2: Photolithographically fabricated Straight and tapered waveguides of a range of widths and taper ratios

receive no electromagnetic interference, and are relatively low cost. Optical interconnects are beginning to penetrate the markets at shorter distances such as in local area networks and as their cost is reduced, will be used within the system enclosure. The use of optics is expected to occur first where the problems for copper are most significant which is for high bit rate, dense interconnections in large area backplanes within non-conducting enclosures. Optical fibres are not the most convenient for interconnections within a system as they can only bend through a large radius of about $10 \mathrm{~cm}$ otherwise light escapes from the fibre core into the cladding resulting in loss and signal corruption. Fibre connectors form a major part of the cost of the optical interconnect and a system with many fibres has many costly connectors. The fibres must be individually routed and errors in routing are time consuming to debug and correct. The fibres can be laid flat on the PCB plane and even bonded together within an epoxy layer, but this is not suited to low cost mass production. An alternative technology suitable for low cost mass production is that of multimode polymer buried channel optical waveguide interconnections within layers in the multilayer PCB formed by the same or slightly modified processes already available within PCB manufacturers. Copper tracks are still required in such substrates to transmit power through the backplane (or motherboard), Figure 1, in order to power mezzanine (or line, or drive, or daughter) boards and copper is still a practical and low cost option at low data rates. Hence there is a need to develop a new type of multilayer hybrid PCB in which optical waveguide interconnects are used for the highest data rates with copper tracks for lower data rates and for power lines and earth planes. These issues have been anticipated by system design companies such as Xyratex Technology, IBM Zurich and Siemens C-Labs, microprocessor designers such as Intel and materials development companies such as Dow Corning, NTT, Rohm and Haas and Exxelis who have instituted research in their own laboratories and in associated universities into optical waveguide interconnect technology. Leading Universities and Research Institutions such as Cambridge (CAPE), University College London (UCL), Heriot Watt University, Loughborough University, National Physical Laboratory (NPL), IMEC - Ghent University, TFCG Microsystems, 
Belgium, Paderborn University, Germany, Helsinki University of Technology, Espoo, Finland and ETRI, South Korea are developing novel polymer materials, developing fabrication techniques, discovering design rules for waveguide layout and carrying out precision characterisation.

Optical buried channel waveguides usually have a core with approximately square or rectangular cross section made from a high refractive index (slow speed of light) material and a cladding surrounding the core of a lower refractive index (higher speed of light). They operate by total internal reflection (TIR) in a similar way to optical fibres. The cost of waveguide connectors is minimised by choosing to use multimode waveguides which typically have cores of $40-70 \mu \mathrm{m}$ width which can tolerate more misalignment than single mode waveguides. The optical buried channel waveguides are formed on a plane by a variety of fabrication techniques which can be implemented, after slight adaptation, in PCB manufacturers. Arrays of low cost vertical cavity surface emitting lasers (VCSELs) emitting $850 \mathrm{~nm}$ wavelength and arrays of photodiodes operating at $10 \mathrm{~Gb} / \mathrm{s}$ are

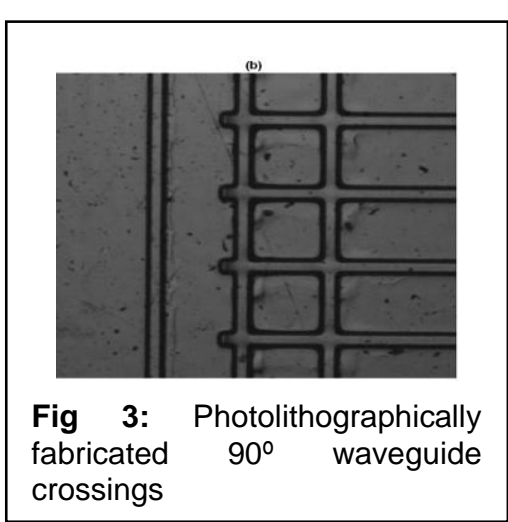
readily available at low cost for use in optical transmitters and receivers. At this wavelength polymer is a convenient low loss material for use as the core and cladding. Polymers can be chosen or designed which can be easily processed to form waveguides at low temperatures, have low cost, and can withstand subsequent high temperature reflow soldering processes.

For optical printed circuit boards to be brought into widespread use, layout tools must be made readily available which design both the copper tracks and the optical waveguides [1]. In 2006 David R. Selviah, UCL formed a large consortium of complementary universities and companies and led a successful bid to carry out a Flagship project entitled "Integrated Optical and Electronic Interconnect PCB Manufacturing (OPCB)" in the Innovative Electronics Manufacturing Research Centre (IeMRC). The consortium companies represented a complete supply and manufacturing chain and route to market for the polymer waveguide technology including companies manufacturing PCB layout tools, computer programs for modelling the behaviour of multimode waveguides, developing and supplying low loss polymer formulations, manufacturing multilayer PCBs, supplying printer fabrication equipment together with end user system companies who require optical printed circuit boards. The following sections describe the project's objectives, the approaches being taken and some examples of what has been achieved so far in the project with an indication of future directions.

\section{The OPCB Project's Objectives}

This three-year research project is exploring novel methods, compatible with traditional multilayer PCB manufacturing processes, for the manufacture of optical waveguides capable of operating at very high data rates within an optical layer in the PCB. Several process routes are under investigation, each with different levels of risk and cost. In addition, modifications are being researched for commercial computer aided design software for PCBs to allow them to also layout optical waveguide patterns. The detailed objectives are:

(a) To establish waveguide design rules for several different manufacturing techniques and to incorporate them into commercial design rule checker and constraint manager layout software for printed circuit boards so that PCB designers can easily incorporate optical connection layers without detailed knowledge of the optics involved. To investigate and understand the effect of waveguide wall roughness and cross sectional shape on the behaviour of light and the effect on waveguide loss.

(b) To develop low cost manufacturing techniques for integrated Optical and Electronic interconnected Printed Circuit Boards, OPCBs. To develop and to compare the commercial and technological benefits of several optical printed circuit board manufacturing technologies photolithography, direct laser-writing, laser ablation, embossing, extrusion and ink-jet printing - for high data rate, small and large (19"), rigid and flexible, printed circuit boards so that it will be clear which technology is best for each type of PCB. To characterise the behaviour of optical waveguide 
backplane systems in real world conditions, including temperature cycling, high humidity and vibration.

(c) To design a commercial, low cost, optical connector (dismountable, passive, self-aligning, midboard) as the next stage from the prototype demonstrated in an earlier project (Storlite Project). To develop novel connector designs suited for interfacing flip chip lasers and photodiodes to OPCBs, and OPCBs to OPCBs through a right angle connector.

\section{The Consortium}

The consortium consists of 3 universities and 10 companies. Dave Milward of Xyratex Technology acts as Industrial Project Manager, while David R. Selviah of UCL is the project Technical Leader. The funding for the project is provided by EPSRC through the IeMRC and by the consortium industrial partners amounting to

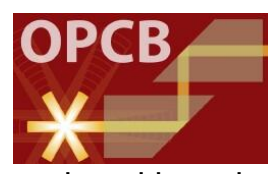
a total of $£ 1.3$ million over 3 years. The EPSRC funding is divided between the three universities who carry out the bulk of the research described in subsequent sections.

\section{Waveguide Layout, Modelling and Characterisation}

University College London (UCL) lay out waveguide test patterns and system demonstrator interconnection patterns using modifications they have made to Cadence software layout tools. Their experimental measurements of these waveguide components after fabrication, Figures 2 and 3 , in various polymers using a range of fabrication techniques are compared to their computer modelled results to gain a detailed understanding of the physical behaviour of coherent light in multimode waveguides and so to establish design rules [2-10]. Figure 4 shows an example of the modelled field in a multimode waveguide bend. A novel theory is being developed for analysing the effects of waveguide side-wall roughness. Low cost, self-alignment techniques are being developed for use in optical connectors for aligning lasers and photodiodes to waveguide end facets and the misalignment tolerances are being assessed. UCL as lead university forges international links with other waveguide researchers such as in their recent mutually beneficial visit to IMEC - Ghent University, TFCG Microsystems, Belgium and promote and disseminate their own [2-10] and the consortiums results [13-14] via a range of international conferences and journal articles.

\section{Laser Direct Writing of Waveguides}

Heriot-Watt University [11,12] has previously developed a direct UV-laserwriting technique so as to form multimode polymer waveguides. In the OPCB project, the key aim is to explore how these techniques can be extended to suit optical backplane applications - both in the context of scale and manufacturability. Fabricating waveguides over metre-scale boards requires not just the ability to write over large areas but also, if production time is to
Fig 4: Computer simulations of the optical field in a $90^{\circ}$ waveguide bend (a) at the start of the bend after a straight input waveguide showing radiated light beyond the outside of the bend, (b) a third of the distance along the bend.

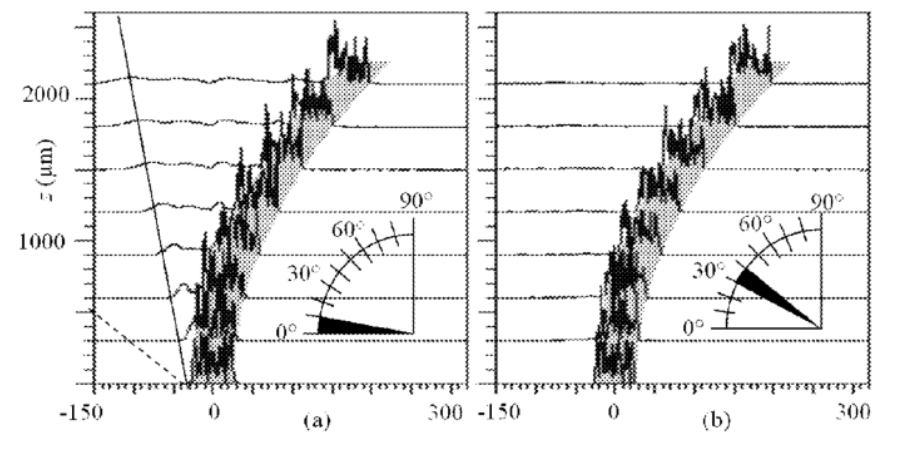


be minimised, faster writing speeds - e.g.> $50 \mathrm{~mm} / \mathrm{s}$. The HWU group has set up a laser-writing facility capable, in principle, of operating over an area $300 \mathrm{~mm} \times 600 \mathrm{~mm}$ at up to 1 metre/sec. Using this system they have been working on the challenge of writing well-defined, low-loss waveguides very much faster than the $100 \mu \mathrm{m} / \mathrm{s}$ write speeds typically used in previous work, Figure 5 . Using laser spots with tailored intensity profiles and an optimised photopolymer formulation, recent results have demonstrated speeds of around $50 \mathrm{~mm} / \mathrm{s}$, for writing $50 \mu \mathrm{m}$ multimode acrylate waveguides. Both straight and curved guides can be fabricated. The HWU group is also exploring fabrication techniques by which their proven techniques for creating embedded $45^{\circ}$ out-of-plane mirrors, Figure, 5 , can be made compatible with large board processing.

\section{Laser Ablation and Inkjet Printing of Waveguides}

Loughborough University are investigating the laser ablation of polymer materials to form waveguides (figure 6). Two routes are being investigated: the first involves the use of a commercial Nd:YAG system based at an industrial partner to machine waveguide structures. This method is attractive as it is utilising equipment already established in PCB manufacturing facilities for the drilling of microvias and therefore would not require additional capital investment. The effect of machining parameters on the depth and speed of ablation is being investigated, together with wall roughness. The second approach considers the use of an excimer laser to form waveguides, however as this type of laser can use a mask projection technique to shape the beam spot, the fabrication of more complex 3D terminations such as curved mirrors at the end of the waveguides is also being investigated.

A significant aspect of the research at Loughborough covers the use of ink-jet printing to deposit polymer waveguide materials. This has the potential to enable fast printing over large areas. For ink jet fabrication, a potential process route is expected to consist of the initial deposition

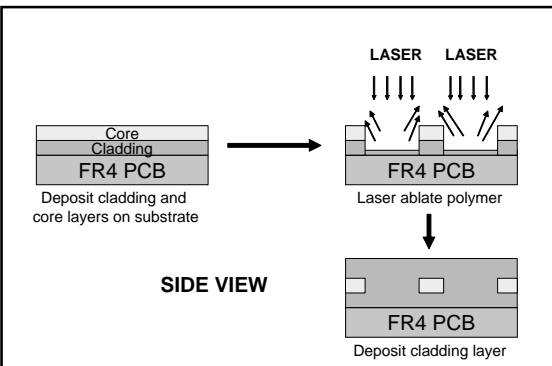

Fig 6: Schematic diagram of laser ablation for the formation of waveguide structures.

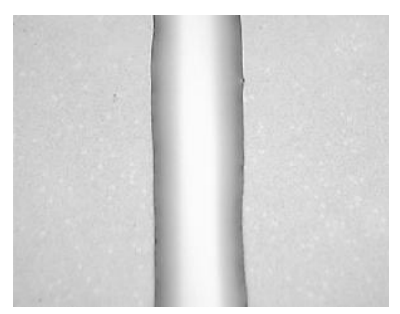

Fig 7: Plan view of ink jet deposited optical waveguide material on a modified glass surface (track is approximately $75 \mu \mathrm{m}$ wide). of a cladding material layer, onto which the core material is jetted to create the appropriate waveguide structure. This will then be enclosed in a further layer of cladding. In order to create core structures with appropriate dimensions and cross-section, the viscosity and surface tension of the jettable core material is crucial in achieving optimum results. Furthermore, the interaction of the ink-jetted material with the substrate is key in determining the wetting behaviour and the stability of the liquid as-deposited structure. Figure 7 shows a line of UV cure optical waveguide material ink jet deposited onto a glass substrate for which surface modification was carried out to control the wetting, enabling a feature approximately $75 \mu \mathrm{m}$ wide and $15 \mu \mathrm{m}$ high to be formed.

\section{Conclusions}

The research being carried out within the IeMRC OPCB project by the 13 member consortium addresses a wide range of problems that need to be solved before multimode polymer waveguide technology becomes widely available. The research ranges from formulation of novel polymers to development of new processes and methods for deposition and patterning of the materials suitable for large area substrates and applicable to PCB manufacturing environments. The characterisation and modelling of these structures will lead to the establishment of design rules for optical waveguide components such as bends and tapers which can be implemented within design and layout software tools, further aiding the uptake of this technology. Further details of the research can be found in [13$16]$. 


\section{Acknowledgments}

The authors thank the UK Engineering and Physical Sciences Research Council for financial support through the Innovative Electronics Manufacturing Research Centre. The technical and financial support of the 10 collaborating companies in the OPCB project is also gratefully acknowledged.

\section{References}

[1] Façanha,H.S., Offen,R.J., Selviah,D.R., Oliveira,C.E.T. (1990). Layout tool for high speed electronic and optical circuits. Layout tool for high speed electronic and optical circuits, London, UK:IEE, 1990/107, 3/1-3.5

[2] Rashed,A.M., Selviah,D.R. (2004). Modelling of Polymer Taper Waveguide for Optical Backplane. Semiconductor and Integrated Opto-Electronics Conference (SIOE'04) Cardiff, UK:SIOE'04, paper 40

[3] Papakonstantinou,I., Selviah,D.R., Fernandez,F.A. (2004). Multimode laterally tapered bent waveguide modelling. LEOS 2004, 17th Annual Meeting of the IEEE Lasers and Electro-Optic Society, Puerto Rico, USA:IEEE, 2, 983-984

[4] Rashed,A.M., Papakonstantinou,I., Selviah,D.R. (2004). Modelling of Polymer Thermo-optic Switch with Tapered Input for Optical Backplane. LEOS 2004, 17th Annual Meeting of the IEEE Lasers and Electro-Optic Society, IEEE LEOS, Puerto Rico:IEEE, 2, 457-458

[5] Rashed,A.M., Selviah,D.R. (2004). Modelling of Polymer 1×3 MMI power splitter for optical backplane. IEEE LEOS Conference on Opto-electronic and Micro-electronic materials and devices, Commad'04, Brisbane, Australia:IEEE, 281-284

[6] Rashed,A.M., Selviah,D.R. (2004). Modelling of the effects of thermal gradients on optical propagation in polymer multimode tapered waveguides in optical backplanes. Photonics North 2004, Software and Modelling in Optics, Ottawa, Canada:SPIE, International Society for Optical Engineering, USA, 5579 (1 and 2), 359-366

[7] Yu,G., Selviah,D.R., Papakonstantinou,I. (2004). Modelling of optical coupling to multimode polymer waveguides: Axial and lateral misalignment tolerance. LEOS 2004, 17th Annual Meeting of the IEEE Lasers and Electro-Optic Society, Puerto Rico, USA:IEEE, 2, 981-982

[8] Papakonstantinou,I., Wang,K., Selviah,D.R., Fernández,F.A. (2006). Experimental Study of Bend and Propagation Loss in Curved Polymer Channel Waveguides for High Bit Rate Optical Interconnections. IEEE Workshop on High Speed Digital Systems, Santa Fe, New Mexico, USA, 1417 May 2006:IEEE

[9] Papakonstantinou,I., Wang,K., Selviah,D.R., Fernandez,F.A. (2007). Transition, radiation and propagation loss in polymer multimode waveguide bends. Optics Express 15(2), 669-679. ISSN: 10944087

[10] Rashed,A.M., Selviah,D.R. (2007). Source misalignment in multimode polymer tapered waveguides for optical backplanes. Optical Engineering 46(1), 015401(1)-015401(7). ISSN: 00913286

[11] McCarthy, H. Suyal and A. C. Walker. "45o Out-of-plane Turning Mirrors for Optoelectronic Chip Carriers based on Multimode Polymer Waveguides." ECOC 2004, Stockholm, Sweden, paper Th1.4.3, (September 5-9, 2004).

[12] A. McCarthy, H. Suyal and A. C. Walker. "Fabrication and Characterisation of Direct LaserWritten Multimode Polymer Waveguides with Out-of-Plane Turning Mirrors." Technical digest of Conference on Lasers and Electro-Optics Europe (CLEO Europe 2005), Munich, Germany, paper Cl14-THU, (June 12-17, 2005).

[13] Selviah,D.R. (2007). Invited Paper: Measurement Challenges for Optical Printed Circuit Boards. Optical Fibre Measurement Conference, OFMC, NPL, Teddington Lock, 15th October 2007, UK, NPL, Teddington Lock, UK

[14]Selviah,D.R., Walker, A. C. and Hutt, D. (2007). Invited Paper: Optical Printed Circuit Boards. leMRC Annual Conference, Henry Ford College, Loughborough, UK September

[15] www.ee.ucl.ac.uk/research/publications

[16] http://www.lboro.ac.uk/research/iemrc/documents/FlagshipProjects/LUW_FSP_01_OPCB.pdf 
